\title{
The Transient Response and Frequency Characteristics of Power Transformers Having Internal Winding Faults
}

\author{
Mohamed M. Saied \\ Professor (Emeritus), Independent Researcher, Giza, Cairo, Egypt \\ Email: mmsaied2@yahoo.com or m.saied@ieee.org
}

\begin{abstract}
A procedure and a corresponding computer program for determining the transient response of a transformer winding under faulty conditions are presented. The model is based on distributed-parameter analysis and is capable of taking the non-uniform distributions of the winding's inductances and capacitances into account. It will be demonstrated that snapshots of the instantaneous voltage and current distributions along the winding at any point in time, as well as the energization response to standard input stimuli can be efficiently utilized for monitoring the winding's conditions. An auxiliary function is suggested for simulating the different kinds, severities and locations of the internal faults. Results for both solidly-earthed and isolated transformer neutral points are given. It will be indicated that the source current includes useful information for assessing the winding's integrity and recognizing the type and location of eventually existing faults. It is believed that the presented approach can assist in providing helpful database for the condition monitoring of power transformers.
\end{abstract}

Keywords: Distributed parameter circuits, condition monitoring, electromagnetic transients, faults, frequency response, integrity, modeling, mutual parameters, non-uniform, power transformers, transient response, winding

\section{Introduction}

There are numerous studies addressing the time- and frequency-domain analyses of power transformers such as those documented in [1-14, 16-19]. Special attention has been devoted to the frequency, and the complex $s$-domain approaches. They have been successfully used in several situations in order to derive closed-form analytical expressions for the voltages and current distributions along the windings and for identifying their resonance frequencies [4-6].

The main approach suggested in reference [7] was the description of the winding by the cascade connection of suitable ladder circuits, followed by the solution of a set of simultaneous differential and algebraic equations. The next step was the application of an alternative concentrated-parameter recursive $s$-domain analytical solution [8].

A more accurate and efficient approach, applying the concept of the frequency- and locationdependent $A, B, C, D$ circuit constants, adopted from the transmission line theory, is presented in $[10$, $11]$.

Reference [12] presents a direct analytical procedure for analyzing windings with non-uniform series inductance, based on the assumption of a quadratic inductance distribution. The analysis of windings exhibiting non-uniform inter-turn insulation capacitance is also given in [13]. An analytical solution is suggested in [14] based on the cascade connection of equivalent transmission lines. Closed-form relations addressing transformers with abruptly changing insulation characteristics are given in [17]. This method could be successfully applied to the more realistic situations involving windings with gradually changing capacitance distributions.

References $[18,19]$ provide a rigorous approach to the simulation of non-uniform windings. It starts with the formulation of two integro-differential equations of the voltage and current. They are expressed in terms of the winding data, the waveform of the applied sources, the neutral point treatment and the complex frequency $s$.

An analytical relation expressing the non-uniformity of the winding's mutual inductive and capacitive couplings is suggested based on the data given in [2]. This leads to a direct solution of the two equations 
utilizing the powerful computer packages such as Mathematica $[15,16]$.

The frequency response analysis is one of the important techniques for the testing and maintenance of power transformers. It can yield key data pertinent to their mechanical integrity. Reference [20] describes in details the possible applications of the Sweep Frequency Response Analysis (SFRA) in monitoring the working conditions of transformers. Useful experimental results of several case studies are presented and discussed. It is considered as one of the best frequency response techniques in this regard. Valuable information about the use of Frequency Response Analysis (FRA) as an efficient tool for detecting the winding mechanical changes and deformation is presented in [21]. The study emphasized the need for more research regarding the sensitivity of each of the presented tests in detecting various fault types. Reference [22] presents a frequency domain technique for the recognition and location of possible axial and radial coil movements as well as eventual mechanical deformations in power transformers. It applies a Laplace domain distributed parameter equivalent circuit resulting from the cascade connection of several two-port networks. The frequency domain transfer admittance is suggested as the main criterion to recognize, identify and locate the winding movement and deformation. The proposed method suggests saving the information about the transfer admittance of the healthy transformer over the frequency range $0-10 \mathrm{MHz}$ as a reference for comparison with the corresponding data during failure.

This paper is a further step in this direction and has the following main objectives:

1. The analysis of the high frequency electromagnetic transients in transformers in the presence of internal winding faults, for various time waveforms of the initiating sources as well as different treatments of the transformer neutral point. The terminal quantities as well as the instantaneous distributions along the faulty winding will be addressed.

2. To investigate the simulation results for different fault types, severities and locations along the winding.

3. To investigate the possibility of using the comparison of the time response during failure with the corresponding data of the healthy transformer as an additional criterion for diagnostics.

4. To explore the possible utilization of these results in identifying the types and locations of these faults.

It is believed that the presented results would lead to useful information regarding the assessment of the winding integrity and fault diagnostics.

\section{Method of Analysis}

\subsection{The Representation of the Healthy Winding}

Consider the representation of the entire transformer winding depicted in Fig.1. A detailed equivalent circuit of an infinitesimal section of length $d x$, in terms of the complex frequency $s$ and the circuit parameters is shown in Fig.2. The section length $d x$ is expressed in per unit based on the winding's entire length. A list of the symbols and the assumed numerical values for the entire winding are given below:

$R$ series resistance $=2.198 \Omega$

$C_{o}$ shunt (earth) capacitance $=20.7 \mathrm{nF}$

$G_{o}$ shunt conductance $=0.15 \mathrm{nS}$

$C_{s}$ series capacitance (mean value $)=2.07 \mathrm{pF}$

Its maximum value is $C_{s} / 0.6934$.

$G_{s}$ winding insulation conductance $=2.07 \mathrm{nS}$

$L_{m}$ the winding's self inductance (mean value) $=0.021 \mathrm{H}$

The maximum value is $L_{m} / 0.6934$.

These parameters are adopted from the references $[8,13]$. 


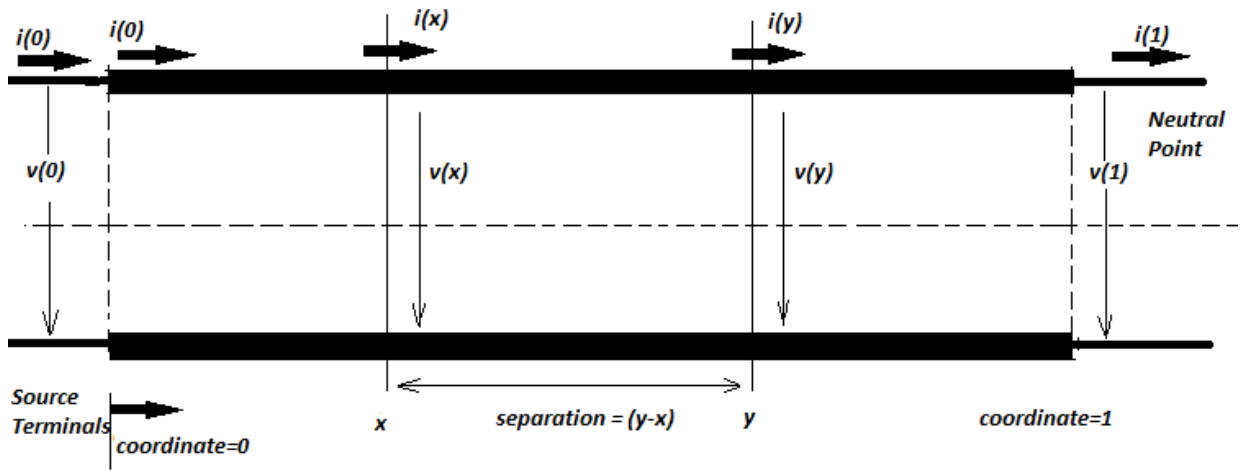

Figure 1. The considered transformer winding

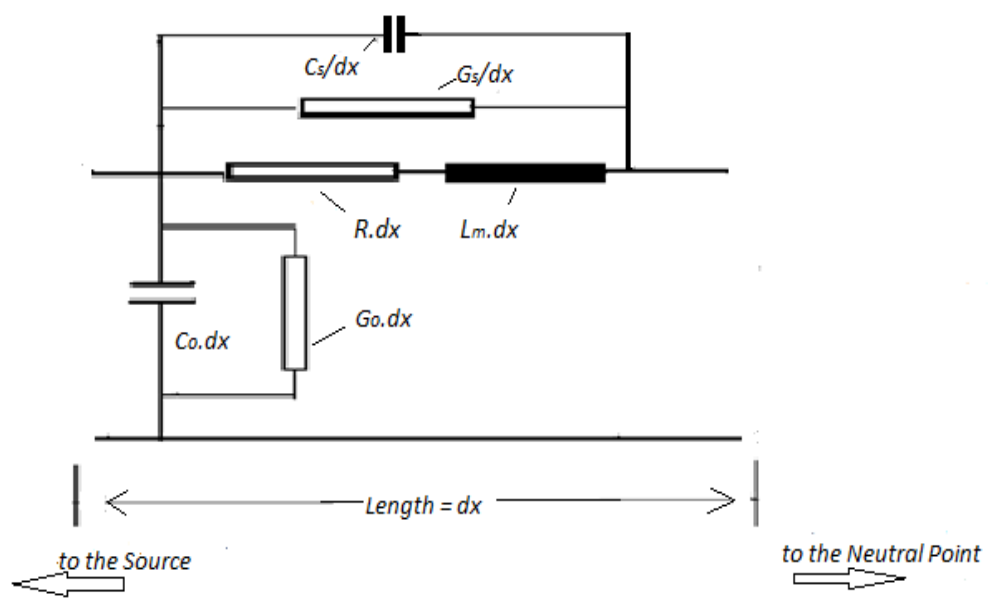

Figure 2. The assumed equivalent circuit for a winding section of length $d x$.

According to the detailed derivations in [18], the winding transients are described by the following two simultaneous equations for the first derivatives of the voltage and current with respect to the co-ordinate $x:$

$$
v^{\prime}(x)=-2.198 i(x)-s \cdot L_{m}\left[\left(1-0.9198 k x^{2}\right) \int_{0}^{1} i(x) d x-0.9198 k \int_{0}^{1} x^{2} i(x) d x+1.8396 k x \int_{0}^{1} x i(x) d x\right]
$$

and

$$
\begin{aligned}
& i^{\prime}(x)=-v(x) \cdot\left(s \cdot C o+G_{O}\right)+ \\
& \left(s \cdot C_{s}+G_{s}\right)\left(\begin{array}{l}
\left(0.9198 k x^{2}-1\right) \cdot v(x)\left(1-0.9198 k x^{2}\right) \\
\left.\int_{0}^{1} v(x) d x-0.9198 k \int_{0}^{1} x^{2} v(x) d x+0.3066 k v(x)-0.9198 k v x(x)+1.8396 k x \int_{0}^{1} x \mathrm{v}(x) \mathrm{d} x\right)
\end{array}\right.
\end{aligned}
$$

for the voltage and current distributions, respectively.

The symbol $s$ denotes the complex frequency, equal to $j \omega$ for the steady state sinusoidal conditions.

The symbol $k$ has the value one if the inter-turn inductive and capacitive couplings are taken into account, or the value $k=0$ otherwise. In all the following case studies, the value $k=1$ was substituted.

It should be noted that the transformer's circuit parameters in the two equations (1) and (2) can generally be functions of the co-ordinate $x$. A direct solution technique is available using the Mathematica software (NDSolve). The proposed direct method requires much less computation burden compared with the numerical solutions. 
Equations (1) \& (2) could be solved by using the Mathematica command (ParametricNDSolve). The output will give the results in the form of two InterpolatingFunctions for the voltages and current at any winding point in terms of its co-ordinate $x$ and the angular frequency $j \omega$. The Laplace or $s$ expressions $v[s, x]$ and $i[s, x]$ can be easily obtained by substituting $\omega=-j s$. More details are available in $[17,18]$. The current and voltage distributions in time domain can then be obtained using one of the available algorithms for numerical inversion of Laplace transform, such as the one of Hosono, as described in [8].

The plots in Figs 3-(a) and 3-(b) depict the input impedance of the healthy transformer winding over the frequency range $0 \leq f \leq 200 \mathrm{kHz}$ and the unit-step response of the source current over the time range $0 \leq t \leq 200 \mu \mathrm{s}$. Both cases of solid-earthing and isolated neutral are displayed. The series and parallel resonance frequencies can be clearly recognized. They are easily determined from the zero crossings of the impedance phase angle as a function of frequency. At these frequencies the input impedance is pure resistive.

In the case of solidly-earthed neutral, the impedance magnitude can reach values as high as $300 \mathrm{k} \Omega$ at the parallel resonance frequencies, and can go below $15 \Omega$ under the series resonance conditions. The first parallel resonance occurs at about $11 \mathrm{kHz}$, while the first series resonance is close to $22 \mathrm{kHz}$. The source current due a unit-step voltage source assumes the initial value of about $1 \mathrm{~mA}$. This implies an initial winding input impedance of approximately $1 \mathrm{k} \Omega$. The current increases continuously with time to its final steady state value close to $1 \mathrm{Volt} / R$ i.e. $456 \mathrm{~mA}$.

Considering the case of isolated neutral point, described by the plots in Fig. 3-(b), the first resonance is a series one occurring at about $11 \mathrm{kHz}$, while the first parallel resonance is close to $22 \mathrm{kHz}$. As expected, the initial source current is $1 \mathrm{~mA}$ which is equal to that of the case of solid earthing. The source current exhibits wave reflections at both the source and neutral terminals. The travel time of these waves along the winding is about $22 \mu \mathrm{s}$. Except for the superimposed higher frequency component, the current curve in Fig. 3-(b) is similar to that of an open circuited conventional transmission line of the same delay time.

The next sections will discuss the sensitivity of these signals to the different types and locations of the winding faults.
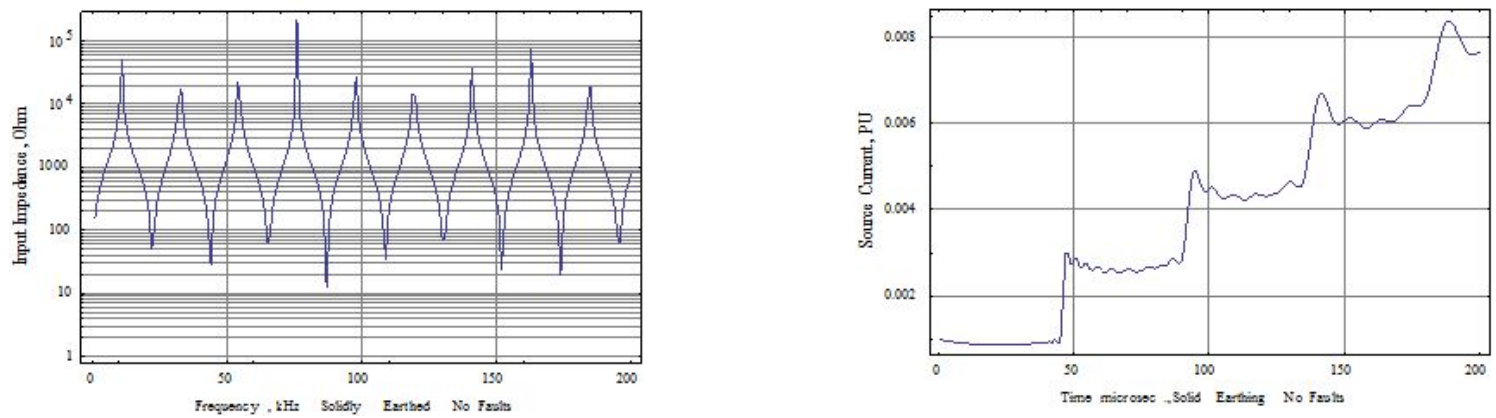

3-(a) The input impedance and the source current unit-step response, solidly-earthed Healthy transformer
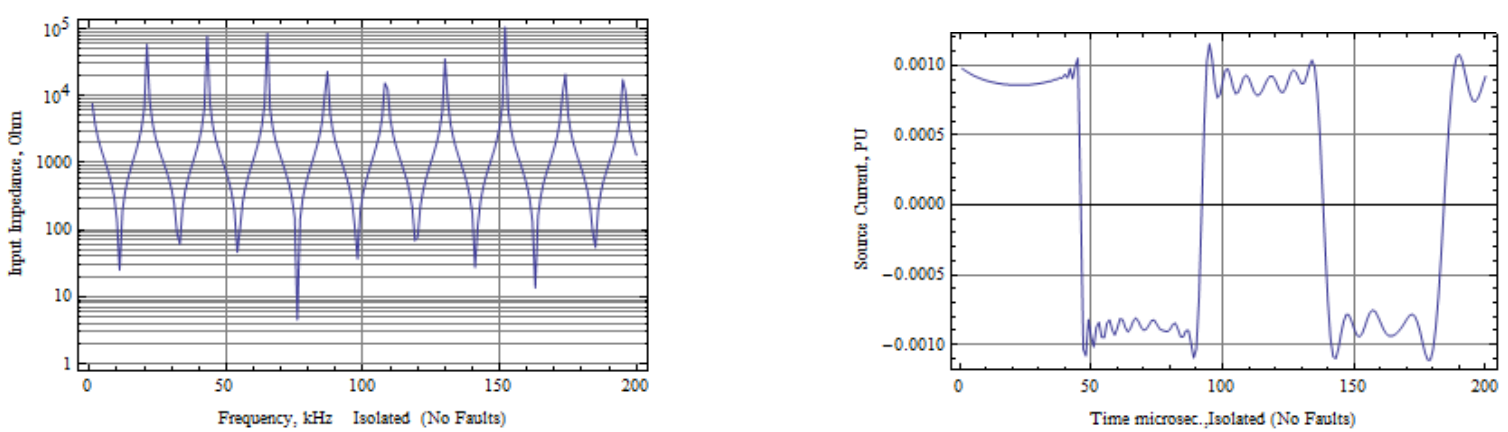

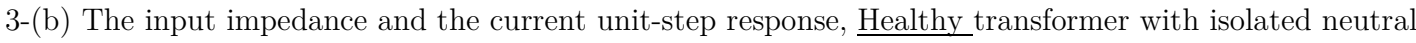

Figure 3. The frequency characteristic of the input impedance and the unit-step response of the source current for both solidly-earthed (a), and isolated neutral (b). Results are for the Healthy transformer. 


\subsection{Representation of the Faulty Transformer}

\section{The Auxiliary Function $f_{a u x}$}

An auxiliary function $f_{a u x}$ of four arguments will be used in order to describe the location (fault) and degree of severity (deg) of the considered fault. The third argument is the per-unit portion of the winding $(2 w)$ affected by the fault. The fourth argument is the variable co-ordinate $(x)$ along the winding. The function should represent a local increase (e.g. a multiplication by deg) in one of the winding's parameters. For instance, Fig.4 shows the auxiliary function describing a fault at a distance fault $=0.6$ per unit measured from the source end. One of the parameters is assumed to increase by the factor 21 , over a winding section having a width $2 w=0.1$ per unit. The appropriate value of the argument $\mathrm{deg}$ in this case is 20 . This function can be formulated as

$$
f_{\text {aux }}=(1+\operatorname{deg}(u[x-(\text { fault }-w)]-u[x-(\text { fault }+w)]))
$$

where $u[x]$ denotes the unit-step function, which is approximated by the expression $\frac{1}{2}\left[\frac{2 \tan ^{-1}(1000 x)}{\pi}+1\right]$. This was done in order to avoid any possible numerical difficulties arising from the discontinuity of the exact unit-step during the solution of the differential equations.

The parameter $d e g$ describes the degree or severity of the considered fault. The special value $d e g=0$ simulates the absence of faults. This leads to $f_{a u x}=1$ over the entire winding.

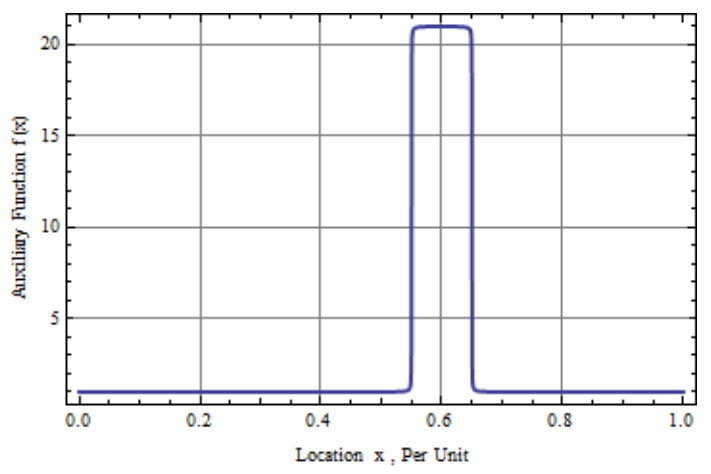

Figure 4. The suggested auxiliary function with the numerical values: fault $=0.60, w=0.05$ per unit and $d e g=20$.

Most of the internal faults can be simulated by multiplying the resistive or conductive elements in the winding's equivalent circuit by a suitable auxiliary function and substitute the result in the coefficients of the two equations (1) and (2). For example, a solid short-circuit or a localized deterioration in the insulation to ground can be expressed by an auxiliary function multiplied by the shunt conductance $G_{0}$. On the other hand, a localized breakdown of the inter-turn winding insulation is simulated by an auxiliary function for the winding insulation conductance $G_{s}$. Similarly, bad contacts can be represented by an auxiliary function for the series resistance $R$. It should be noted that this procedure allows for multiple simultaneous faults of different kinds at different locations.

\section{$3 \quad$ Sample Results}

\subsection{A Solid Short Circuit at $\mathrm{x}=0.8$ per Unit}

A comparison of the plots in Fig.5 with the corresponding ones in Fig.3-(a) indicates higher values for the series and parallel resonance frequencies. This can be attributed to the $20 \%$ shorter active part of the winding, leading to an approximately $20 \%$ shorter delay time and to correspondingly higher resonance frequencies. The input impedance at the parallel resonance frequencies is generally smaller than those of the healthy transformer. The opposite is true for the impedance values at the series 
resonance frequencies. Moreover, a faster increase of the source current is noticed in the case of the faulty transformer. This is due to the fact that the equivalent circuit in this case is dominated by the series inductance. The initial value of the source current is again $1 \mathrm{~mA}$, unaffected by the fault. It should be noted that, with the solid shunt fault, the plots will not be affected by the neutral impedance, as expected.
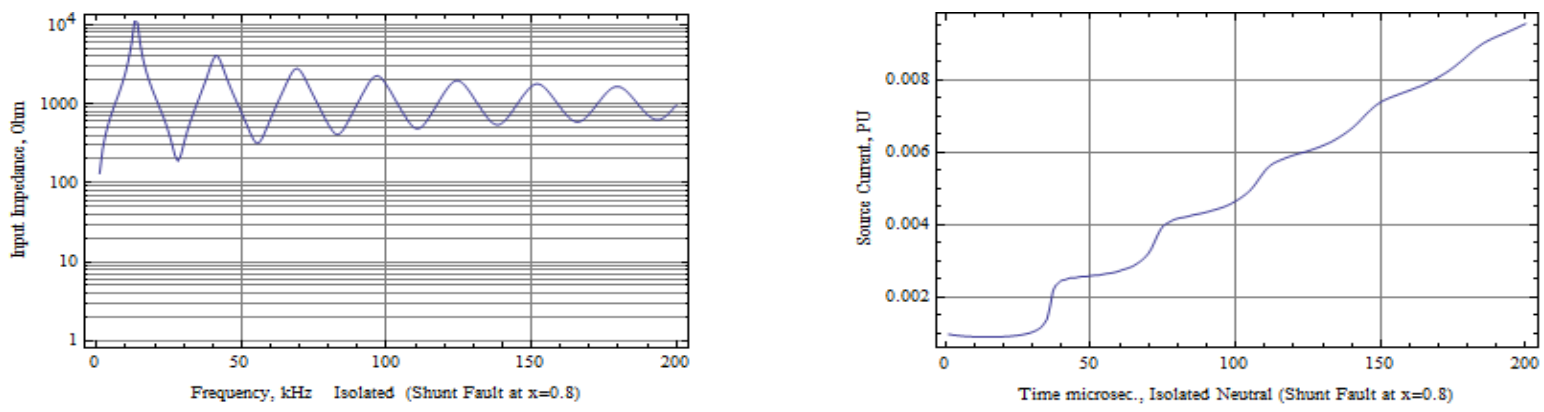

Figure 5. The frequency characteristic of the input impedance (left) and the unit-step response of the source current (right) which are identical for both cases of solidly-earthed and isolated neutrals. There is a shunt fault at $x=0.8$ per unit.

\subsection{Series Fault at $\mathrm{x}=\mathbf{0 . 8}$ per Unit}

The case of a loose connection or conductor open-circuit can be analyzed using an auxiliary function affecting the winding's series resistance per unit length R. Again here, there is no difference between the plots of the cases of solid-earthing or open neutral.
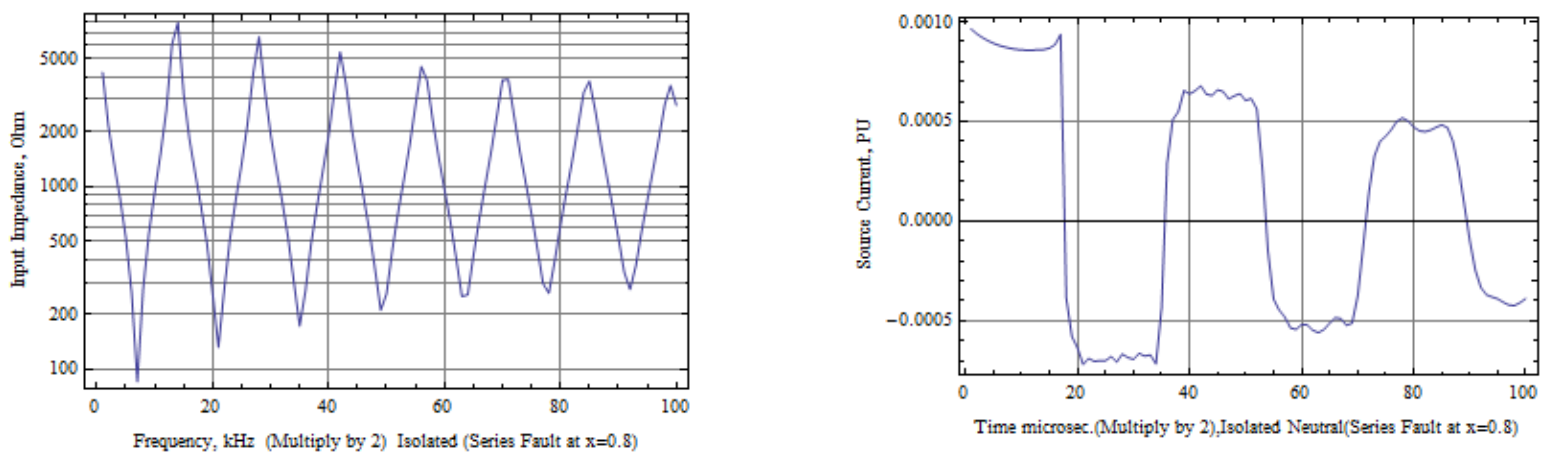

Figure 6. The frequency characteristic of the input impedance and the unit-step response of the source current which are identical for both cases of solidly-earthed and isolated neutral. There is an open-circuit fault at $x=0.8$ per unit.

It is noticed that the resonance frequencies are higher than those of the healthy winding depicted earlier in Fig.3-(b) addressing the case of isolated neutral. The $20 \%$ shorter delay time of the line section between the source and the fault location can be observed by inspecting the corresponding curves of the source current. As expected, the initial value of the source current, which depends only on the source voltage and the winding's input impedance at the time point $t=0$, remains the same $(1 \mathrm{~mA})$. It can be further seen that the source current is approaching its steady state value of zero.

\subsection{Surface Faults}

This section addresses faults resulting from a possible deterioration in the turn-to-turn insulation. In this type of faults, several winding turns, and accordingly a considerable faulty winding section (defined earlier as $2 w$ ), can be involved. Fig. 7 shows the results for a surface fault covering a winding section of a width $2 w=0.20$ per unit extending from $x=0.4$ to $x=0.6$ per unit. The neutral point is assumed isolated. 
The value $d e g=10^{12}$ is substituted in the expression of the auxiliary function. Smaller values can be used for a partial breakdown of the insulation. For simulating this type of faults, an auxiliary function is multiplied by the winding insulation conductance $G_{s}$.
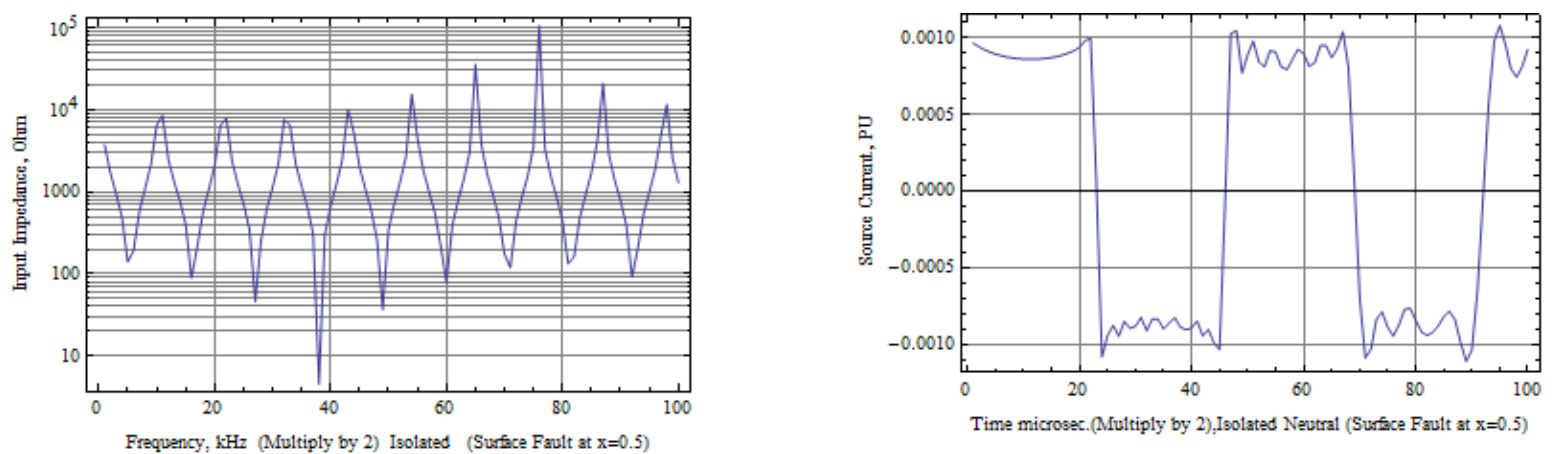

Figure 7. The frequency characteristic of the input impedance and the unit-step response of the source current for a faulty winding with isolated neutral. There is a surface fault at $x=0.5$ per unit.

Since the number of the winding's turns is unaffected by this type of fault, the resonance frequencies depicted in Fig.7 are identical to those of the healthy transformer shown in Fig. 3-(b). The higher losses due to surface fault will only reduce the sharpness of the impedance peaks at resonance.

\subsection{Windings with Simultaneous Faults}

Two or more auxiliary functions can be incorporated in the two equations (1) and (2) in order to simulate simultaneous internal faults. For instance, if an auxiliary function for the winding insulation conductance $G_{s}$ with the location fault $=0.5$ per unit and another one for the shunt conductance $G_{o}$ having also fault $=0.5$ per unit (not necessarily equal), the results of a surface fault and a simultaneous shunt fault will be obtained. They are illustrated in Fig. 8 for the case of isolated neutral. The impedance resonance frequencies are generally higher compared with those in Fig.3-(b) for the healthy winding with isolated neutral. Moreover, their number within the considered frequency range $0 \leqslant f \leqslant 200 \mathrm{kHz}$ is fewer. The current starts with the initial vale of $1 \mathrm{~mA}$, which is common in all cases, and then increases rapidly to about $130 \mathrm{~mA}$ after 200 usec. The initial steps in the current curve are due to the wave reflections and they imply a winding's delay time of about $11 \mu \mathrm{sec}$, which is almost half the corresponding value under healthy conditions, as expected. The higher losses introduced by the simultaneous faults lead to an increase in the impedance values at the series resonance frequencies. There is also a reduction in the impedance at the parallel resonances.
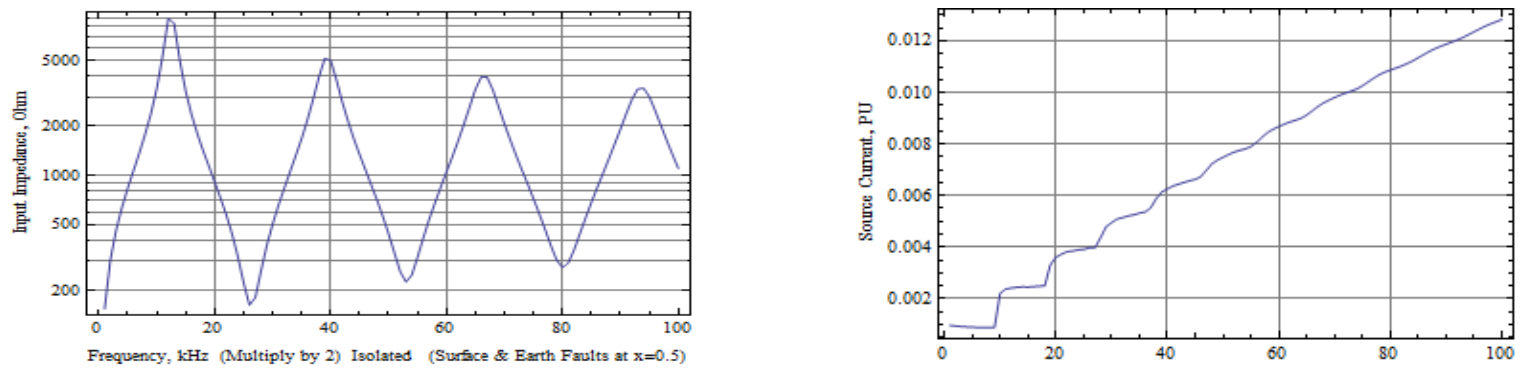

Time microsec.(Multiply by 2),Isolated Nevtral (Sufface \& Earth Favilts at $x=0.5$ )

Figure 8. The input impedance and the source current of a faulty winding with isolated neutral and two simultaneous faults, both at $x=0.5$ per unit 


\subsection{The Internal Current and Voltage Distributions}

In order to demonstrate the capability of the suggested technique to analyze the internal current and voltage distributions, snap shots for both quantities, 100us after applying the unit-step voltage source, are depicted in Figs. 9 and 10, respectively. They refer to the case of two simultaneous faults at $x=0.5$ and $2 w=0.2$ addressed in Fig.8. The impact of the faults is much clearer in the current distributions. The instantaneous current values drop from about $0.0075 \mathrm{~mA}$ directly before the fault location $x=0.5$ per unit to approximately $0.0002 \mathrm{~mA}$ directly after the fault. This decrease occurs gradually over the faulty section of width $2 w$, i.e. in the range $0.4 \leq x \leq 0.6$ per unit.
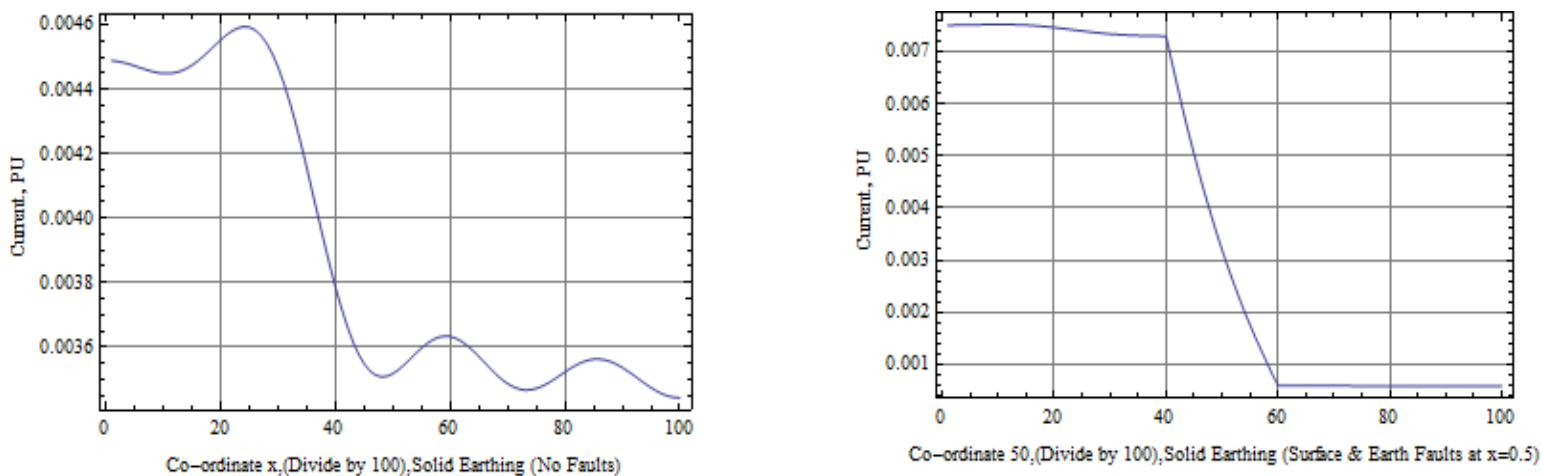

Figure 9. The current distribution along the winding at $t=100 \mu$ s for the healthy winding (left) and the faulty winding (right). neutral is solidly-earthed.
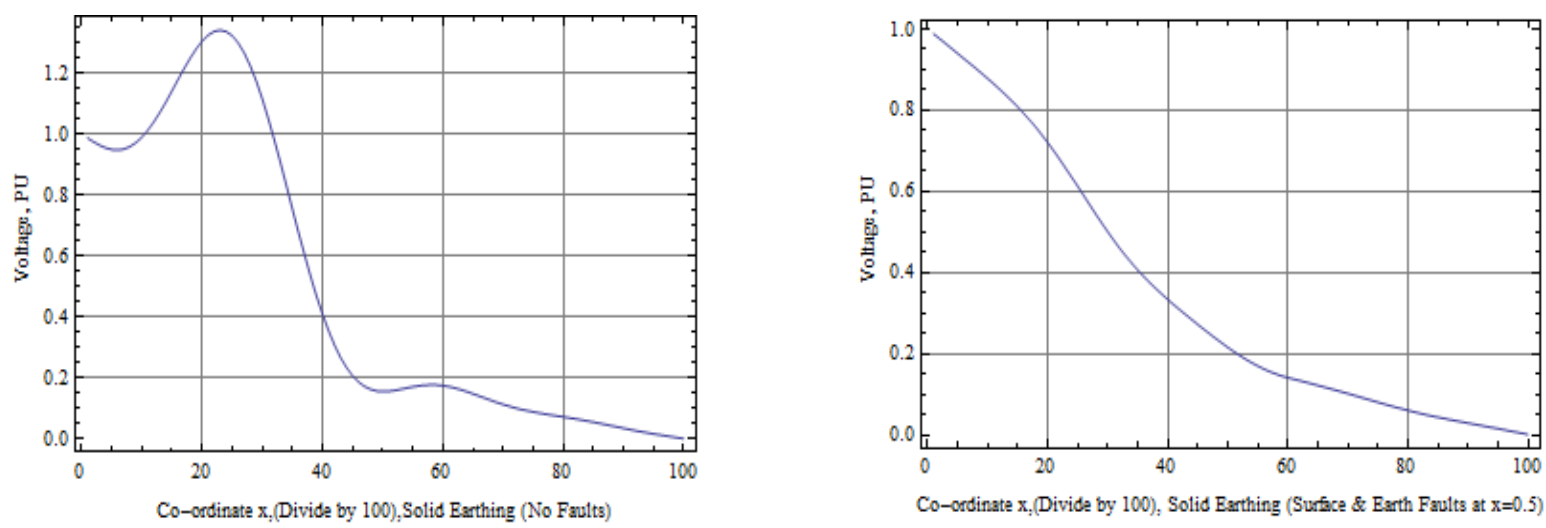

Figure 10. The voltage distribution along the winding at $t=100 \mu \mathrm{s}$ for the healthy winding (left) and the faulty winding (right). neutral is solidly-earthed.

The voltage distribution along the healthy solidly-earthed winding at $t=100 \mu$ s exhibits a local voltage increase (about 1.35 per unit) at the winding point of the co-ordinate $x=0.24$ per unit. This instantaneous value decreases to 0.65 per unit in the presence of the two simultaneous faults.

\section{Conclusions}

A procedure is presented for analyzing power transformer windings in the presence of internal faults. The frequency characteristics and the transient response can be determined. The inter-turn inductive and capacitive couplings are taken into account. The suggested model can handle any time waveform of the input source initiating the transients and any connection of the transformer neutral point. Auxiliary functions are suggested for simulating the different fault types, severities and locations. Situations involving simultaneous faults at different winding points can also be dealt with. Sample results of different case studies are presented and discussed. In addition to the impact of the faults on the terminal 
quantities such as the transient input source current and the voltage of eventually existing neutral points, the voltage and current distributions along the faulty winding at any point in time can also be determined. It is believed that the presented procedure as well as the ease of its implementation will yield helpful information regarding the assessment of the winding's integrity and possible fault diagnostics.

\section{References}

1. A. Greenwood. Electrical Transients in Power Systems, Book, Wiley-Interscience, Second edition, 1991, Chapter 11.

2. R.C. Degeneff. A General Method for Determining Resonances in Transformer Windings, IEEE Trans. on PAS, Vol.96, 1977, No.2, pp.423-430

3. S. Hosseini, M. Vakilian, G. Gharehpetian. Comparison of Transformer Detailed Models for Fast and Very Fast Transient Studies, IEEE Trans. Power delivery, Vol.23, no.2 pp.733-741, April 2008.

4. M. M. Saied, A.S. AlFuhaid. Electromagnetic Transients in Line-Transformer Cascade by a Numerical Laplace Transform Technique, IEEE Trans. on PAS, Vol.104, 1985, pp.2901-2909

5. M.M. Saied, A.S. AlFuhaid. Frequency Response of Two-Winding Transformers Obtained by a DistributedParameter s-Domain Method, Journal of Electric Power Components and Systems, Vol.32, 2004, No.8, pp. 755766.

6. R. Jayaratchagan, B. Shriram. Impulse Voltage Distribution, International Journal of Engineering Research \& Technology (IJERT), and Vol.2 Issue 4, April - 2013, ISSN: 2278-0181, pp. 1203-1207.

7. M.M. Saied. New Solution Technique for the Frequency and Transient Response of Transformer Windings with All Inter-Turn Mutual Inductances and Capacitances Included, Paper No. 3143, J. Trends In Electrical Engineering, Volume 3, Issue 1, ISSN: 2249-4774, 2013.

8. M.M. Saied. A Contribution to the Frequency Analysis and the Transient Response of Power Transformers' Windings, J. Electric Power Components and Systems, 42:1143-1151, 2014

9. M. Popov, L. Sluis, R. Smeets. Complete analysis of Very Fast Transients in Layer-type Transformer Windings, Presented at the International Conference on Power Systems Transients (IPST'07) in Lyon, France on June 4-7, 2007

10. M. M. Saied. Transformer Modeling for The Frequency and Transient Analyses, with Non-Uniform Inductance Emulating The Inter-Turn Magnetic Coupling, Journal Electrical Power and Utilization, (EPQU) Vol XVII, No.1, pp. 1-11, 2014.

11. A. Predota, Z. Benesova. Analysis of Transients in Transformer Winding Respecting Space-Varying Inductance, PRZEGLĄD ELEKTROTECHNICZNY (Electrical Review), ISSN 0033-2097, R. 88 NR 7b/ (2012), pp. $220-222$.

12. M. M. Saied. Modeling of Transformer Windings having Non-uniform Inductance Distribution, J. Of Power Electronics \& Power Systems, Vol.3, Issue3, pp. 12-25, 2014.

13. A. Predota, Z. Benesova, L.Koudela. Fast Transient Overvoltage in Transformer Winding, Przegląd Elektrotechniczny (Electrical Review), ISSN 0033-2097, R. 87 NR 5/ (2011), pp. 142-145.

14. M. M. Saied. The Transient Response and Frequency Characteristics of Power Transformers Having NonUniform Winding Insulation, J. Of Power Electronics \& Power Systems, Vol.4, Issue 1, pp. 37-52, (2014).

15. Wolfram Research. Documentation on the software Mathematica 10, available at: http://www.wolfram.com/mathematica/?source=nav , 2014

16. Wolfram Mathematica Tutorial Collection. Advanced Numerical Differential Equation Solving in Mathematica, Book, Wolfram Research Inc., 2008, available at:

http://www.wolfram.com/learningcenter/tutorialcollection/AdvancedNumericalDifferentialEquationSolvingInMat hematica/

17. M. M. Saied. An Analytical Method for the Analysis of Transformer Windings Having Non-Uniform Capacitance Distribution, Journal of Power Electronics \& Power Systems, Vol.5, Issue2, pp. 1-14, 2015.

18. M. M. Saied. Frequency Characteristics of Transformer Windings with Separation-Dependent Inter-Turn Mutual Parameters, Journal of Power Electronics \& Power Systems, Vol.6 (2016), Issue 6.pp. 72-81.

19. M. M. Saied. Transients in Transformers with Non-Uniform Parameter Distributions, Submitted for publication in Journal Electrical Power and Utilization, (EPQU), 2017. 
20. C. Sweetser, T. McGrail. Sweep Frequency Response AnalysisTransformer Applications, A Technical Paper from Doble Engineering, Tech Paper 01.doc, Version 1.0 01/27/03, pp.1-3, 2003.

21. P. Picher (Convener). Mechanical-condition monitoring assessment of transformer windings using Frequency Response Analysis (FRA), Cigre Tutorial of Working Group WG A2.26

22. M. M. Saied, M. A. Al-Shaher. Recognition of Power Transformer Winding Movement and Deformation Using FRA, Int. Journal of Computation and Mathematics in Electrical and Electronics Engineering (COMPEL), 26 (5), pp. 1392-1409. 